\title{
Tratamento de águas residuárias da indústria de laticínios por meio de wetland construído
}

\section{Samara Pereira Vieira ${ }^{1}$, Anderson Brito da Silva ${ }^{1}$, Síntia da Silva Freire $^{1}$ e Luiz Antonio Pimentel Cavalcanti ${ }^{2}$}

${ }^{1}$ Instituto Federal de Educação. Ciência e Tecnologia da Bahia. Campus de Paulo Afonso. Rua Marcondes Ferraz, 200. Bairro General Dutra. Paulo Afonso-BA, Brasil (CEP 48607-000).

${ }^{2}$ Instituto Federal de Educação, Ciência e Tecnologia da Bahia. Curso de Ensino Básico, Técnico e Tecnológico (EBTT). Campus de Paulo Afonso. Rua Marcondes Ferraz, 200. Bairro General Dutra. Paulo Afonso-BA, Brasil (CEP 48607-000). E-mail: luiz.cavalcanti@ifba.edu.br.

Resumo. Os wetlands construídos estão entre as tecnologias mais eficientes recentemente comprovadas para tratamento de águas residuárias. Em comparação aos sistemas convencionais já existentes, destaca-se pelo baixo custo e facilidade de operação e manutenção. Com base nisto, o presente trabalho consistiu em dimensionar e construir um protótipo de wetlands, a fim de realizar o tratamento de águas residuárias da indústria de laticínios visto que a mesma traz consigo uma quantidade elevada de carga orgânica. A vegetação escolhida para compor o sistema foi a baronesa Eichhornia crassipes (Mart.) Solms (Commelinales: Pontederiaceae), pois, além de ser a espécie flutuante mais utilizada para esse tipo de tratamento, também vem causando grandes problemas com a poluição de rios e lagos do Município de Paulo Afonso, Estado da Bahia, Nordeste do Brasil. Para o experimento foram recolhidos $100 \mathrm{~L}$ de efluente para um período de nove dias no qual foram recolhidas amostras do efluente bruto e após o tratamento objetivando realizar um comparativo. As variáveis físicas e químicas avaliadas foram $\mathrm{pH}$, turbidez, DQO, e sólidos dissolvidos, comparadas com os parâmetros estabelecidos pela Resolução CONAMA no 430/2011. O pH aproximou-se ao padrão estabelecido, e houve uma atenuação significativa de turbidez (91\%), DQO (89\%) e sólidos dissolvidos (92\%). Os resultados obtidos após a análise constataram a eficiência do protótipo de wetland construído com a utilização da baronesa como alternativa para redução de impactos causados pelo efluente de laticínios.

Palavras-chave: Tratamento de água; Wetlands construídos; Macrófitas; Baronesa.

Abstract. Treatment of wastewater from the dairy industry by means of constructed wetland. The wetlands are among the most recently proven technologies for wastewater treatment. Compared to existing conventional systems it stands out for the

Recebido:

25/05/2019

Aceito:

20/07/2019

Disponível on line:

$21 / 07 / 2019$

Publicado:

31/08/2019

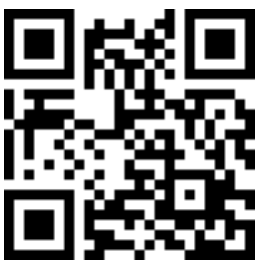

Acesso aberto

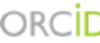

(ㄷ) 0000-0001-5491-8720 Caio Fernando da Silva

D 0000-0001-5915-2007

Flávia Maria Torres

Lima

D 0000-0002-4870-7238

Flávia Maria Torres

Lima

D) 0000-0003-4932-9387

Luiz Antonio Pimentel Cavalcanti 
low cost and ease of operation and maintenance. Based on this, the present work consisted in designing and constructing an wetlands prototype in order to carry out the wastewater treatment of the dairy industry as it carries with it a high amount of organic load. The vegetation chosen to compose the system was the common water hyacinth (Eichhornia crassipes (Mart.) Solms (Commelinales: Pontederiaceae), because, besides being the most used floating species for this type of treatment, it is also causing great problems with the pollution of the rivers and lakes of the Municipality of Paulo Afonso, Bahia State, Northeast Brazil. For the experiment, $100 \mathrm{~L}$ of effluent were collected for a period of 9 days in which samples were collected from the raw effluent and after the treatment, aiming at comparing the tables. The physical and chemical variables evaluated were $\mathrm{pH}$, turbidity, COD, BOD and dissolved solids compared to the parameters established by Resolution 430, May 6, 2011, of the Brazilian National Environment Council (CONAMA). The pH approached the established standard, and there was a significant attenuation of turbidity (91\%), COD (89\%) and dissolved solids (92\%). The results obtained after the analysis verified the efficiency of the wetland prototype constructed with the use of the common water hyacinth as an alternative to reduce the impacts caused by the effluent of dairy products.

Keywords: Water treatment; Constructed wetlands; Macrophytes; Common water hyacinth.

\section{Introdução}

0 crescente aumento populacional e industrial impactam diretamente nas mudanças climáticas associadas à emissão de gases de efeito estufa que por sua vez afetam os processos hidrológicos, como a disponibilidade e a qualidade de água potável (doce). Essas mudanças tendem aumentar a temperatura e, consequentemente, a evaporação com a provável redução da disponibilidade hídrica podendo dessa forma, induzir riscos ambientais, econômicos e sociais. Tendo em mente que a mesma representa meramente $3 \%$ de toda água do planeta e que está cada vez mais poluída é de suma importância que sejam buscadas alternativas no sentido de conservar e preservar esse bem natural (Liang, 2017).

Com a alavancada do setor industrial de laticínios nos últimos anos é natural que surja uma preocupação em termos ambientais visto que é um setor com grande utilização da água em todo o processo industrial. Essa água utilizada traz consigo uma quantidade elevada de resíduos sólidos concentrados, bem como uma elevada carga orgânica. Tal fenômeno aponta para a necessidade de se encontrar formas de tratamento que levem, não somente à minimização desses resíduos, como também à devolução de uma água que não coloque em risco outros corpos d'água atendendo a Resolução CONAMA no 430/2011 (Siqueira, 2014).

É certo que algumas empresas já se preocupam em buscar alternativas nesse sentido, pode-se citar o sistema convencional de lagoas como um desses mecanismos. Porém, atualmente os sistemas de tratamento do tipo wetlands são vistos pela comunidade cientifica como uma alternativa viável economicamente para locais com amplas áreas, e tem como principais atrativos a independência de energia elétrica para o processo, bem como, a não necessidade 
de produtos químicos além forte apelo ambiental e possibilidade de compor o paisagismo local (Silva Junior e Almeira, 2015).

Em termos de caracterização os wetlands construídos são réplicas feitas pelo homem dos wetlands naturais que refina a exploração dos ciclos biogeoquímicos que normalmente acontecem nos sistemas de tratamento de águas residuais. Que inclui a criação de um habitat para fauna e flora. Existem diferentes tipos que se diversificam com base na característica de fluxo da água e espécies vegetais. E pode ser implantado onde a água residuária é gerada (Reis e Serbent, 2015; Silva et al., 2018).

\section{A Eichhornia crassipes (Mart.)}

Solms (Commelinales: Pontederiaceae) também conhecida como baronesa está entre as espécies flutuantes mais utilizadas no mundo para tratamento de águas residuárias e para despoluição dos rios (Siqueira, 2014). E com base nisso foi a vegetação escolhida para compor o protótipo de wetlands, visto que $\mathrm{o}$ Município de Paulo Afonso-BA vem sofrendo o aparecimento contínuo nos rios e lagos, de modo que a prefeitura e autoridades locais estão em busca constante de formas de mitigar esse problema.

Com base nisso o presente trabalho teve como objetivo dimensionar e construir um protótipo de wetlands construídos no intuito de realizar $o$ tratamento de águas residuárias de laticínios e que garanta o seu descarte conforme os parâmetros estabelecidos pela Resolução CONAMA no 430/2011 (Brasil, 2011), bem como de fornecer ao Município de Paulo Afonso uma alternativa para o surto de baronesas que vem comprometendo os rios e lagos da cidade.

\section{Materiais e métodos}

Dimensionamento do protótipo

O dimensionamento do protótipo foi realizado considerando o efluente residual da produção de laticínios e foi equacionado conforme os seguintes parâmetros:

\section{Volume do reator}

$$
\mathrm{V}=\mathrm{a} \cdot \mathrm{b} \cdot \mathrm{c} \quad \text { Equação } 1
$$

Onde a, b e c são as dimensões de um recipiente paralelepipédico.

\section{Tempo de detenção hidráulica}

É o tempo médio (geralmente expresso em dias) em que os despejos líquidos permanecem em uma unidade ou sistema.

$$
t=n \frac{V}{Q} \quad \text { Equação } 2
$$

Onde:

t é o tempo de detenção hidráulica (dia);

$\mathrm{n}$ a porosidade do leito filtrante $\left(\mathrm{m}^{3}\right.$ vazios $/ \mathrm{m}^{3}$ material);

$$
\begin{aligned}
& \mathrm{V} \text { o volume do leito }\left(\mathrm{m}^{3}\right) ; \\
& \mathrm{Q} \text { a vazão a ser tratada }\left(\mathrm{m}^{3} / \mathrm{d}\right) .
\end{aligned}
$$

Constante de reação cinética de primeira ordem

Pode ser obtida por equações empíricas que relacionam a constante de reação a $20{ }^{\circ} \mathrm{C}\left(\mathrm{K}_{20}\right)$ com a equação modificada de Van't Hoff-Arrhenius:

$$
\begin{aligned}
& K_{T}=K_{20} \cdot(1,06)^{T-20} \quad \text { Equação } 3 \\
& \begin{array}{c}
\text { Onde } \mathrm{T} \\
\text { operação. }
\end{array}
\end{aligned}
$$

\section{Área do wetland construído}

$$
\begin{aligned}
& A=\frac{Q \cdot(\ln C o-\ln C e)}{K_{T} \cdot n \cdot H} \quad \text { Equação } 4 \\
& \text { Onde: } \\
& \text { Q é a vazão a ser tratada; } \\
& \text { Co a concentração afluente em }
\end{aligned}
$$
termos de $\mathrm{DBO}_{5}(\mathrm{mg} / \mathrm{L})$;

Ce é a concentração efluente em termos de $\mathrm{DBO}_{5}(\mathrm{mg} / \mathrm{L})$;

$\mathrm{K}_{\mathrm{T}}$ a constante de reação da cinética de primeira ondem, dependente de $\mathrm{T}\left(\mathrm{d}^{-1}\right)$;

$\mathrm{H}$ a altura da coluna d'água do reator. 


\section{Construção do protótipo}

Para a construção do protótipo utilizou-se uma caixa d'água com capacidade de $100 \mathrm{~L}$ para armazenar o efluente bruto. Um organizador transparente de plástico com $0,2035 \mathrm{~m}^{2}$ como leito para o tratamento do efluente e quatro recipientes de $20 \mathrm{~L}$ para coletar o efluente tratado de modo que a medida que fosse enchendo o recipiente fosse sendo substituído e dessa forma possibilitando o acompanhamento do tratamento, tendo em vista o tempo de retenção hidráulica já pré-determinado como mostrado nos cálculos de dimensionamento. Para as tubulações foram utilizadas uma vara de tubo PVC $20 \mathrm{~mm} \times 6 \mathrm{~m}$, cinco curvas de $90^{\circ}$, um flange, um tê, dois tampões e dois registros do tipo esfera, todos de material PVC com $20 \mathrm{~mm}$. A Figura 1 mostra a construção da tubulação no leito de tratamento.

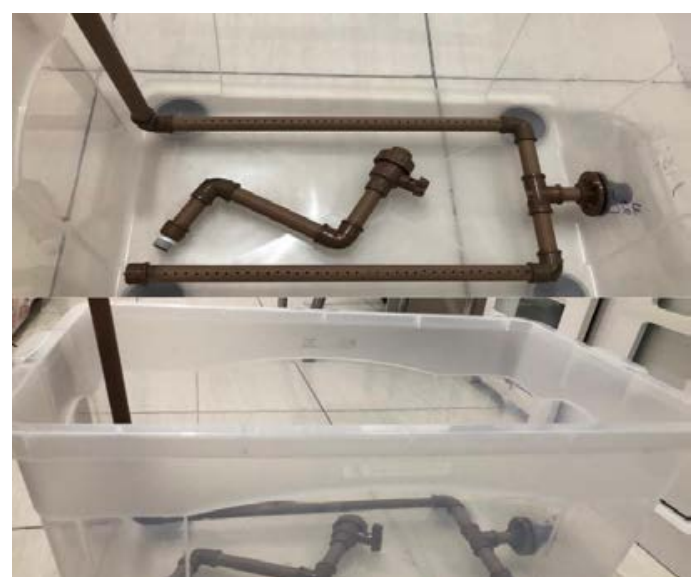

Figura 1. Construção do prototipo de Wetlands para tratamento de águas residuais.

\section{Filtro de leito fixo}

O filtro de leito fixo foi composto por uma camada areia seguida de uma camada de brita que na qual foi posicionada a tubulação de distribuição do efluente bruto. Esse filtro será responsável por reter os poluentes do efluente. Logo abaixo do filtro estará posicionada a tubulação de coleta do efluente tratado.

\section{Vegetação}

Indivíduos de E.crassipes, também conhecidos como baronesas, foram recolhidos no Rio São Francisco, no Município de Paulo Afonso, Estado da Bahia. Por se tratar de uma espécie flutuante deixamos um espaço no leito para que pudesse encher com água bruta e garantir a permanência das plantas em meio flutuante.

Tratamento de efluente residual de laticínios através de wetlands construídos

0 efluente residual foi recolhido em uma fábrica de laticínios localizada nas proximidades do Município de Paulo Afonso, Estado da Bahia. O volume de $100 \mathrm{~L}$ do efluente foram utilizados para realizar o tratamento que perdurou nove dias. Para análise foram recolhidas duas amostras sendo elas do efluente bruto e do último dia de tratamento para que pudesse ser realizado um comparativo do efluente de antes e após o tratamento no que tange aos parâmetros estabelecidos pela Resolução CONAMA no 430/2011 para qualidade de despejo de águas residuárias (Brasil, 2011).

\section{Determinação do pH}

Os valores de $\mathrm{pH}$ das soluções brutas e pós-tratamento das águas residuais foram medidos via pHmetro digital (Quimis, modelo: Q400AS) a $25^{\circ} \mathrm{C}$.

\section{Determinação da turbidez}

As medidas de turbidez foram realizadas via método nefelométrico (Eaton et al., 2005), em turbidímetro de bancada (Turbidímetro multiprocessado DLM 2000B, Del Lab®). 


\section{Determinação de DQO}

As análises de DQO foram realizadas via método colorimétrico (Standard Methods 5220 D) com bloco digestor do tipo TE-021 DryBlock Digestor (TECNAL). A DQO nas amostras foi quantificada por espectrofotometria (Spectrophotometer SP1105, Bel Photonics), tomando-se como branco um padrão água destilada (BioClass) (Eaton et al., 2005).

\section{Resultados e discussão}

\section{Protótipo de sistema de wetland construído horizontal}

Realizada uma extensa revisão da literatura com intuito de estudar os componentes e equipamentos a fim de ter uma base necessária para a construção do sistema de Wetland Construído, foi alcançado os cálculos aplicando as equações citadas na metodologia e obteve-se o volume do reator 40,7 x 10-3 $\mathrm{m}^{3}$ de acordo com a Equação 1, o tempo de detenção hidráulica foi de 3,44 dias, para uma vazão de $8,285 \times 10^{-3} \mathrm{~m}^{3} /$ dia, Equação 2, com temperatura média de Paulo Afonso em torno de $35{ }^{\circ} \mathrm{C}$, tendo como área do Wetland 0,20349 $\mathrm{m}^{2}$, utilizando a Equação 3. À vista disso, por meio dos cálculos efetuados, foi possível a construção do protótipo e posterior avaliação do tratamento. A Figura 2 mostra o protótipo de wetlands em funcionamento, construído com base em todas as etapas anteriormente descritas.

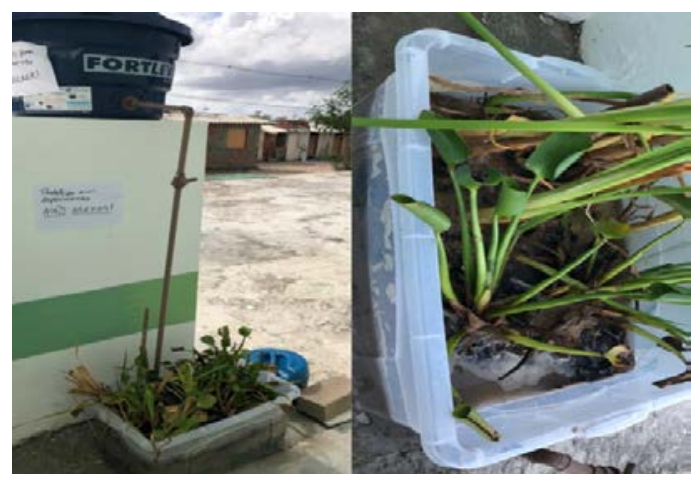

Figura 2. Tratamento de água residual de laticínios via wetlands construídos.

\section{Análise das variáveis}

A Tabela 1 apresenta os valores das variáveis contidas na água residual de laticínio antes e após o tratamento realizado no wetland construído. Nota-se a eficiência da remoção da carga orgânica no sistema cultivado com a E. crassipes da região.

Tabela 1. Comparação de valores das variáveis medidas do efluente antes e após tratamento.

\begin{tabular}{lrr} 
Variável & Bruto & Tratado \\
\hline $\mathrm{pH}$ & 3,78 & 4,67 \\
Turbidez (UNT) & 79,88 & 7,04 \\
Condutividade $(\mu \mathrm{S} / \mathrm{cm})$ & 992,76 & 89,32 \\
DQO $(\mathrm{mg} / \mathrm{L})$ & $5.145,54$ & 565,95 \\
Sólidos dissolvidos & $1.716,45$ & 137,28 \\
\hline
\end{tabular}

Com base nos resultados obtidos após o tratamento do efluente, observouse um decréscimo das variáveis medidas, classificando-o em qualidade básica para o descarte. $0 \mathrm{pH}$ aproximou-se ao padrão estabelecido, e houve uma diminuição significativa de turbidez (91\%), DQO (89\%) e Sólidos Dissolvidos (92\%), sendo esses bastante discutidos na literatura.

\section{pH}

Inicialmente $\mathrm{o}$ afluente inserido no wetland foi caracterizado ácido. Neste sentido, Quege (2011) afirma que o pH diminui à medida que os vegetais e 
microorganismos desenvolvem-se, de maneira que muitas bactérias responsáveis pelo tratamento de resíduos líquidos sobrevivem dentro de uma faixa de $\mathrm{pH}$ entre 4,0 e 9,5, pois há um bom desenvolvimento bacteriano na neutralidade. Entretanto, apesar de a água residual aplicada ter $\mathrm{pH} 3,78$, próximo a 4,0, o sistema demonstrou-se capaz de tamponar o meio e produzir um efluente com $\mathrm{pH}$ predominantemente ácido 4,67, próximo ao valor indicado pela Resolução CONAMA no 430/2011 para lançamento em curso d'água, entre 5 e 9. Dessa forma, é possível, considerando essa diferença, que não tenha havido interferência direta na variação do $\mathrm{pH}$.

\section{(DQ0)}

\section{Demanda química de oxigênio}

Os dados indicam que foram obtidos bons resultado na redução de DQO em consequência da influência significativa das plantas e também da filtração atuando como um biofilme. Os resultados podem ser considerados pelo tempo de detenção hidráulica no wetland. Uma vez que a E.crassipes (baronesa) é bastante estudada por uma grande capacidade de crescimento vegetativo, já notado no Balneário Prainha de Paulo Afonso-BA (Figura 3), é usada no protótipo por ser uma forma ecológica de tratamento de efluentes industriais e sanitários como um sistema biológico (Coelho, 2017).

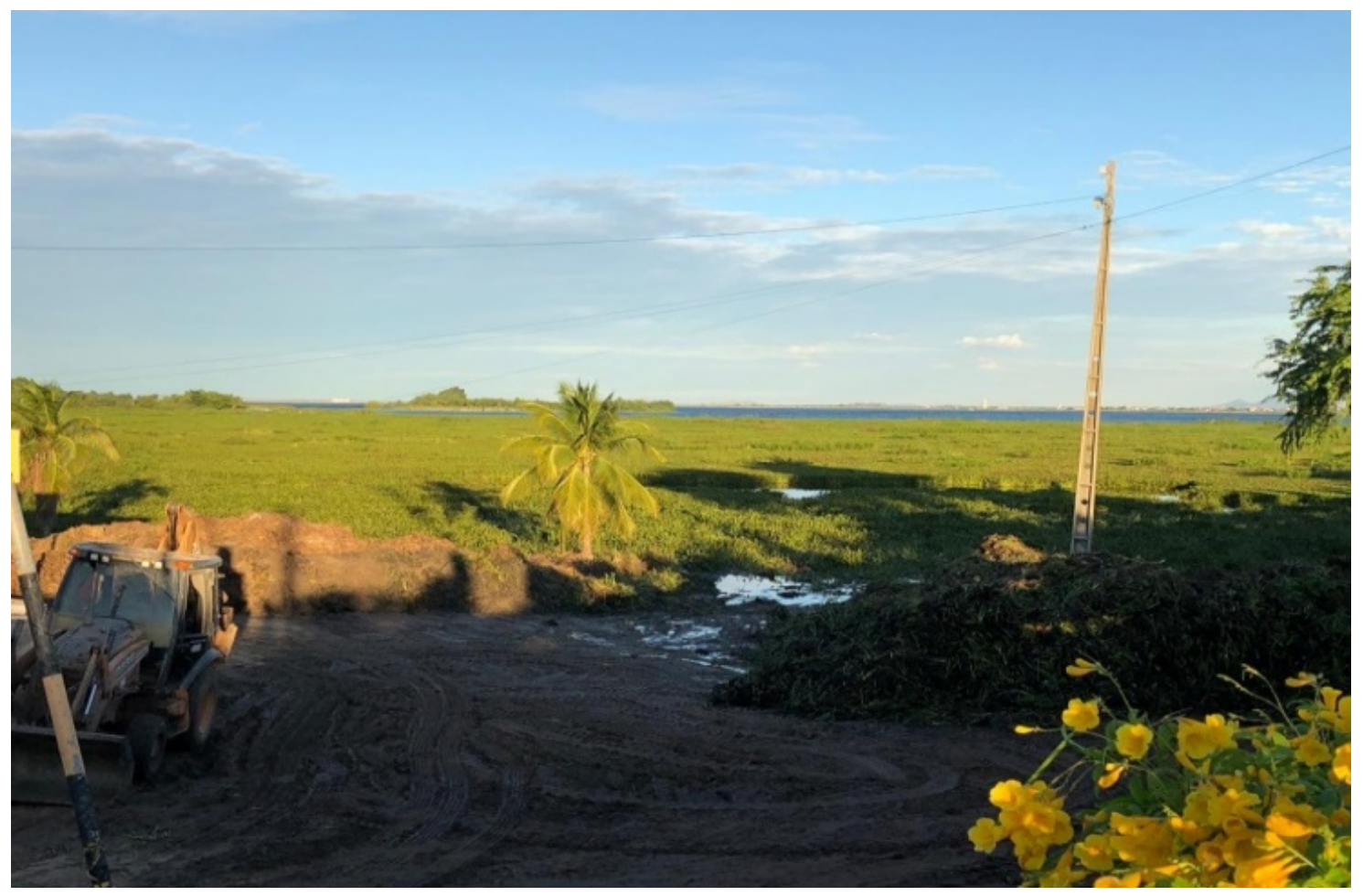

Figura 3. Vista do Balneário Prainha de Paulo Afonso-BA infestado de E. crassipes (baronesas).

De maneira geral, wetlands construídos operam de forma eficiente, visto essa considerável redução de DQO, também da redução de turbidez e sólidos dissolvidos. Tais resultados corroboram com os bons resultados apresentados na literatura. Como salienta Costa et al. (2013), os wetlands são uma boa alternativa tecnológica para a remoção da carga orgânica e dos sólidos em 
suspensão. Além disso, mostra o desempenho da baronesa como protagonista, posto que é capaz de absorver e fixar em seus tecidos elementos em alta quantidade e absorver altos teores de metais pesados (Coelho, 2017). Desta forma, sugere-se a comunidade a aplicação desse sistema a fim controlar a eutrofização que atrai essa espécie e a considera como uma praga.

\section{Conclusão}

O dimensionamento do protótipo de wetlands mostrou boa eficiência constatada pelos resultados obtidos no efluente residual de laticínios tratado com o pH próximo ao padrão estabelecido, e uma diminuição significativa de turbidez (91\%), DQO (89\%) e sólidos dissolvidos (92\%) no qual se enquadrou nos parâmetros estabelecidos na Resolução CONAMA no $430 / 2011$ e dessa forma podendo ser descartada sem oferecer riscos a outros corpos d'água. Os resultados obtidos após a análise constataram a eficiência do protótipo de wetland construído com a utilização das baronesas como alternativa para redução de impactos causados pelo efluente de laticínios, além de trazer alternativa para as autoridades locais da cidade de Paulo Afonso-BA para solucionar o impacto causado pela grande quantidade das espécies que vem ocupando os rios e lagos da região.

\section{Conflito de interesses}

Os autores declaram não haver conflito de interesses.

\section{Referências}

Brasil. Resolução CONAMA no 430, de 13 de maio de 2011. Dispõe sobre as condições e padrões de lançamento de efluentes, complementa e altera a Resolução no 357, de 17 de março de 2005, do Conselho Nacional do Meio Ambiente-CONAMA. Disponível em: <http://www2.mma.gov.br/port/conama/le giabre.cfm?codlegi=646>. Acesso em: $24 \mathrm{abr}$. 2019.

Coelho, J. C. Macrofitas aquáticas flutuantes na remoção de elementos químicos de água residuária. Botucatu: Faculdade de Ciências Agronômicas, UNESP, 2017. (Dissertação de mestrado).

Costa, J. C.; Paoli, A. C.; Seidl, M.; Sperling, M. Performance and behaviour of planted and unplanted units of a horizontal subsurface flow constructed wetland system treating municipal effluent from a UASB reactor. Water Science and Technology, v. 68, n. 7, p.1495-1502, $2013 . \quad$ https://doi.org/ 10.2166/wst.2013.391

Eaton, A. D.; Clesceri, L. S.; Rice, E. W.; Greenberg, A. E.; Franson, M. A. H. (Ed.). Standard methods for the examination of water and wastewater. 21. ed. Washington: American Public Health Association, 2005.

Kadlec, R. H.; Wallace, S. Treatment wetlands. 2. ed. Boca Raton, Florida: CRC Press, 2008.

Liang, Y.; Zhu, H.; Bañuelos, G.; Yan, B.; Zhou, Q.; Yu, X.; Cheng, X. Constructed wetlands for saline wastewater treatment: A review. Ecological Engineering, v. 98, p. 275-285, 2017. https://doi.org/10.1016/j.ecoleng. 2016.11.005

Macedo, J. A B. Águas e águas. São Paulo: Varela, 2001.

Quege, K. E. Sewage treatment by root zone system using bamboo plants. Goiânia: Universidade Federal de Goiás, 2011. (Dissertação de mestrado).

Reis, A.; Serbent, M. P.; Rodrigues, E. B. Wetlands construídos como alternativas para o tratamento de efluentes em unidades de conservação. Revista Brasileira de Gestão Ambiental e Sustentabilidade, v. 2, n. 3, p.137-144, 2015. Disponível em: <http://revista.ecogestaobrasil.net/v2n3/v0 2n03a06a.html>. Acesso em: 24 abr. 2019.

Salati, E. Controle de qualidade de água através de sistemas wetland construídos. Rio de Janeiro: Fundação Brasileira para o Desenvolvimento Sustentável, 2010.

Silva C. S.; Ramos, M. L. G.; Bernardes, R. S. Remoção de sais em wetlands construídos no tratamento de esgotos domésticos primário. Gesta - Revista Eletronica de Gestão e Tecnologias Ambientais, v. 6, n. 1, 
p. 125-138, 2018. https://doi.org/10.9771/ gesta.v6i1.22371

Silva Junior, E. D.; Almeida, R. A.; Siqueira, E. R.; Silva, A. R.; Rodrigues, A. M. Tratamento de resíduos sépticos em wetlands construídos de fluxo vertical. Anais do II Simpósio Brasileiro sobre Wetlands Construídos, Curitiba, 2015.

Siqueira, E. R. Tratamento dos resíduos de fossas e tanques sépticos em um sistema de alagado construído. Goiânia: Escola de Engenharia Civil, Universidade Federal de Goiás, 2014. (Dissertação de mestrado).

Von Dreifus, T. Tratamento de águas residuárias por alagados construídos de fluxo subsuperficial horizontal, utilizando vetiver (Chrysopogon zizanoides L.): avaliação e desempenho de três leitos distintos. Bauru, São Paulo: Universidade Estadual Paulista-UNESP, 2012. (Dissertação de mestrado). 